International Journal of Difference Equations (IJDE).

ISSN 0973-6069, Volume 16, Number 1, (2021). 95-105

(C) Research India Publications

https://dx.doi.org/10.37622/IJDE/16.1.2021.95-105

\title{
Enhanced Cuckoo Intelligence Search Algorithm
}

\author{
${ }^{1}$ Ibukun Isaac Aina, ${ }^{1}$ Olumuyiwa James Peter, ${ }^{2}$ Abayomi Ayotunde Ayoade, ${ }^{3}$ \\ Festus Abiodun Oguntolu, ${ }^{4,5}$ Matthew Olanrewaju Oluwayemi \\ ${ }^{1}$ Department of Mathematics, University of Ilorin, Ilorin, Kwara State, Nigeria \\ ${ }^{2}$ Department of Mathematics, University of Lagos Nigeria, Lagos State, Nigeria \\ ${ }^{3}$ Department of Mathematics, Federal University of Technology Minna, Niger State Nigeria \\ ${ }^{4}$ Landmark University SDG 4 (Quality Education Research Group), Omu-Aran, Nigeria. \\ ${ }^{5}$ Department of Mathematics, Landmark University, P.M.B. 1001, Omu-Aran, Nigeria.
}

\begin{abstract}
Cuckoo Search (CS) algorithm is a meta-heuristic technique that displays several merits. For example, it is easier to apply and less tuning parameters also, it is suitable for solving optimization problems. However, easily fall into local optimum has been established and has a slow convergence rate as a result of the cuckoo search parameters being kept constant. Therefore to handle this issue, an Enhanced Cuckoo Intelligence Search (ECIS) algorithm was developed which is an upgraded CS algorithm. The efficiency of ECIS was tested by some benchmark constrained optimization test functions and it was shown that ECIS gives a better optimal value than CS.
\end{abstract}

Keywords: Cuckoo search algorithm, enhanced cuckoo intelligence search algorithm and levy flight.

\section{INTRODUCTION}

CS is built on the life of a bird named 'cuckoo' [1]. The rudimentary of this unique optimization technique is the laying of egg and breeding of this bird. In this modeling we use the egg of adult cuckoos and the cuckoos. The adult cuckoos eggs are being laid in other birds territory. Then the development of the egg occur and the become a mature cuckoo if they are not detected and detached by the owner of the nest(host birds). The migration of collections of cuckoos and environmental conditions expectantly lead them to congregate and reach the optimum place for breeding and reproduction. 
CS was designed by Yang and Deb [1]. The CS algorithm was stirred by the parasitic reproductive behavior of some cuckoo class by egg laying their host nest. This decreases the likelihood of the eggs being ignored so re-productivity rises. It is significant to indicate that several host birds involve in direct conflict with interfering cuckoos. In general, hatching of cuckoo egg is a bit earlier than that of their host. When the first egg of the cuckoo is hatched which brings the first cuckoo chick, his first disposition action is to eject the egg of its host by sightlessly forcing the eggs of the host outside the nest. This deed leads to growing the cuckoo chick's share of food that the host bird provide. The breeding conduct of cuckoo can be well implemented in several optimization problems. Lévy Flights tool is used instead of ordinary random walk to improve the efficiency [2].

[3], proposed a modified cuckoo search algorithm by introducing random inertia weight $\mathrm{C}$ algorithm parameter in order to improve the peed of convergence and accuracy of the CS algorithm. [4], proposed a multimodal CS algorithm which is a modification of the standard CS algorithm. This was done by introducing the memory mechanism, new selection strategy and depuration procedure. Other works on modified cuckoo search and some other metaheuritic algorithms can be found in [5], [6], [7], [8], [9].

To the best of our knowledge, we have identified that no work has been done on cuckoo search by updating the cuckoo search parameter $\left(p_{a}, \phi\right.$ and $\left.\alpha\right)$ at each iteration. We therefore complement and extend previous works by considering $p_{a i}, \phi_{i}$ and $\alpha_{i}$ which is the value of $p_{a}$ of the iteration, value of $\phi$ of the current iteration and value of $\alpha$ of the current iteration respectively. The aim of this work is to reduce the possibility of the CS algorithm being trapped by local optimal solution by modifying the fixed cuckoo search algorithm parameters. The motivation behind this work is the premature convergence of CS algorithm.

The rest of the paper is arranged as follows: Chapter two deals with experiment and discussion, chapter three deals with computational consideration, chapter four deals with discussion on numerical results and chapter five deals with the conclusion.

\section{EXPERIMENT AND DISCUSSION}

The parameter switching $\left(p_{a}\right)$ and step size $(\alpha)$ used in CS help the technique to generate an improved solution.

These parameters are major in fine-tuning of solution and are used in the modification of speed of convergence of the algorithm. 


\subsection{Pseudo code of CS Algorithm}

Data: Objective function $f(x)$

Result: Best or optimal solution

Step 1: Initialization of parameters $\left(n, p_{a}, \phi\right.$ and $\left.\alpha\right)$

Step 2: Generate initial population of $n$ host nest $x_{i}$

Step 3: While ( $\mathrm{t}<$ MaximumGeneration) or (stop criterion) do $t=t+1$

Step 4: Randomly get a cuckoo search

Step 5: Generate solution by Levy flights

Step 6: Evaluate its objective $f_{i}$

Step 7: Choose randomly a nest among $n$ (say, $j$ )

Step 8: If $\left(f_{i}>f_{j}\right)$ then replace $j$ by the new solution $i$

Step 9: End

Step 10: A fraction $\left(p_{a}\right)$ of worse nests are abandoned

Step 11: Now nests/solution are generated

Step 12: Keep best solution(or nests with quality solutions)

Step 13: Rank the solutions and find the current best solution

Step 14: Go to step 3

Step 15: End.

\subsection{Parameter CS Algorithm}

The below are the parameters used in the above Cuckoo Search(CS) Algorithm Levy flight:

$$
L(s, \phi)=\frac{\phi \Gamma(\phi) \sin (\pi \phi / 2)}{\pi s^{1}+\phi}, \quad 0 \leq \phi \leq 2
$$

$\phi$ is always chosen to be 1.5

Step size:

$$
\alpha=0.01\left(x_{i}-x_{\text {best }}\right) s
$$


where

$$
s=\frac{u}{|v|^{1 / b e t a}}, \quad u=\left(0, \sigma^{2}\right), v=(0,1)
$$

where $u$ and $v$ are random number drawn from normal distribution

$$
\sigma=\left[\frac{\Gamma(1+\phi) \sin (\pi \phi / 2)}{\phi \Gamma[(1+\phi) / 2] 2^{(\phi-1) / 2}}\right]^{1 / \phi}
$$

where $\Gamma$ is the standard gamma function. In practice its easier to choose $\sigma=0.01$ update of nest(global search):

$$
x_{i}^{t+1}=x_{i}^{t}+\alpha * L(s, \phi)
$$

update of nest(local search):

$$
x_{i}^{t+1}=x_{i}^{t}+\alpha s * H\left(p_{a}-\epsilon\right)\left(x_{j}^{t}-x_{k}^{t}\right)
$$

where $*$ is an entry-wise multiplication and $x_{j}^{t}$ and $x_{k}^{t}$ denotes two different solutions randomly selected by random permutation.

$H(u)$ is a heaviside function define as a unit step discontinuous function in which its value is zero and one for non negative number selected from a uniform distribution.

\subsection{Enhanced Cuckoo Intelligence Search (ECIS) Algorithm}

The parameter $p_{a}, \phi$ and $\alpha$ introduce in the CS algorithm enhance the global and local solution of the algorithm. The parameters are significant in fine-tuning of solution vectors, and in adjusting the rate of convergence of the algorithm, it can be potentially used.

For $p_{a}, \phi$ and $\alpha$ fixed value are used in CS algorithm. These values are set at the initial step of the algorithm and cannot be changed during new generations. This main weakness of this techniques appears in the number of iteration to find an optimum.

In this paper a new method is proposed, this method is an enhanced CS called enhanced Cuckoo Intelligence Search (ECIS) Algorithm which implement calculating or generating a new value of $p_{a}, \phi$ and $\alpha$ at each iteration or at every generation.

In this algorithm we introduce and implement the following:

We replace $p_{a}, \phi$ and $\alpha$ by $p_{a i}, \phi_{i}$ and $\alpha_{i}$ respectively in the cuckoo search algorithm, where

$$
\begin{aligned}
p_{a_{i}} & =p_{a_{\max }}-\frac{i}{i t_{\max }}\left(p_{a_{\max }}-p_{a_{\min }}\right) \\
\phi_{i} & =\phi_{\max }-\frac{i t_{e r}}{i t_{\max }}\left(\phi_{\max }-\phi_{\min }\right)
\end{aligned}
$$




$$
\alpha_{i}=\alpha_{\max }-\frac{1}{i t_{\min }} \ln \left(\frac{\alpha_{\min }}{\alpha_{\max }}\right)
$$

Tab 1:Parameter of Enhanced Cuckoo Intelligence Search (ECIS) Algorithm and meaning

\begin{tabular}{|c|c|}
\hline \hline Parameter & Meaning \\
\hline iter & number of current iteration \\
\hline$i t_{\max }$ & maximum number of iteration \\
\hline$p_{a_{i}}$ & value of $p_{a}$ of current iteration \\
\hline$p_{a_{\min }}$ & minimum value of $p_{a}$ \\
\hline$p_{a_{\max }}$ & maximum value of $p_{a}$ \\
\hline$\phi_{i}$ & value of $\phi$ of current iteration \\
\hline$\phi_{\min }$ & minimum value of $\phi$ \\
\hline$\phi_{\max }$ & maximum value of $\phi$ \\
\hline$\alpha_{i}$ & value of $\alpha$ of current iteration \\
\hline$\alpha_{\min }$ & minimum value of $\alpha$ \\
\hline$\alpha_{\max }$ & maximum value of $\alpha$ \\
\hline
\end{tabular}

Various test show that suitable algorithm parameter leading to good result can approximately be values in table 2.2

Tab 2:Value for Parameter of enhanced Cuckoo Intelligence Search (ECIS) Algorithm

\begin{tabular}{|c|c|}
\hline \hline Parameter & Value \\
\hline$p_{a_{\min }}$ & 0.005 \\
\hline$p_{a_{\max }}$ & 1.0 \\
\hline$\phi_{\min }$ & 2.0 \\
\hline$\phi_{\max }$ & 1.0 \\
\hline$\alpha_{\min }$ & 0.5 \\
\hline$\alpha_{\max }$ & 0.05 \\
\hline
\end{tabular}

\subsection{Pseudo Code of Enhanced Cuckoo Intelligence Search (ECIS) Algorithm}

Data: Objective function $f(x)$

Result: Best or optimal solution

Step 1: Initialization of parameters $\left(n, p_{a_{\max }}, p_{a_{\min }}, \phi_{\max }, \phi_{\min }, \alpha_{\max }, \alpha_{\min }\right)$

Step 2: Generate initial population of $n$ host nest $x_{i}$

Step 3: While ( $\mathrm{t}<$ MaximumGeneration) or (stop criterion) do

$t=t+1$ 
Step 4: Calculate $p_{a_{i t e r}}, \phi_{i t e r}, \alpha_{i t e r}$

Step 5: Get a cuckoo randomly

Step 6: Generate solution by Levy flights

Step 7: Calculate its solution quality or objective $f_{i}$

Step 8: Select a nest among $n$ (say, $j$ ) randomly

Step 9: If $\left(f_{i}>f_{j}\right)$ then replace $j$ by the new solution $i$

Step 10: End

Step 11: A fraction $\left(p_{a}\right)$ of worse nests are abandoned

Step 12: New nests/solution are built/generated

Step 13: Keep best solution(or nests with quality solutions)

Step 14: Rank the solutions and find the current best solution

Step 15: Go to step 3

Step 16: End.

\section{COMPUTATIONAL CONSIDERATION}

The numerical results are presented in table 4.1 and it was obtained from the solution of some test problems presented in this section using MATLAB R2010a (7.10.499) run on the PC Intel(R) samsung, a 32 bit Os Laptop windows 7 operating system.

The following conventional benchmark problems from [10], were used to test the performance of the new (ECIS) algorithm:

$F 1=5 h_{1}+5 h_{2}+5 h_{3}+5 h_{4}-\sum_{c=1}^{4}-\sum_{c=5}^{4} h_{c}^{13} h_{c}$

subject to

$$
\begin{aligned}
& q(1)=2 h(1)+2 h(2)+h(10)+h(1) \leq 10 \\
& q(2)=2 h(1)+2 h(3)+h(10)+h(12) \leq 10 \\
& q(3)=2 h(2)+2 h(3)+h(11)+h(12) \leq 10 \\
& q(4)=-8 h(1)+h(10) \\
& q(5)=-8 h(2)+h(11) \\
& q(6)=-8 h(3)+h(12) \\
& q(7)=-2 h(4)-h(5)+h(10) \\
& q(8)=-2 h(6)-(7)+h(11) \\
& q(9)=-2 h(8)-h(9)+h(12) \\
& h(c)=[0,1], \quad c=1, . ., 9 \\
& h(c)=[0,100], \quad c=10,11,12 \\
& h(13)=[0,1]
\end{aligned}
$$


$F 2=5.35785472 h(3)^{2}+0.8356891 h(1) h(5)+37.293239 h(1)-40792.141$

subject to

$q(1)=0 \leq 85.334407+0.0056858 h(1) h(4)+0.0006262 h(1) h(4)-0.0022053 h(3) h(5) \leq 92$

$q(2)=90 \leq 80.51249+0.0071317 h(2) h(5)+0.0029955 h(1) h(2)+0.0021813 h^{2}(3) \leq 110$

$q(3)=20 \leq 9.300961+0.0047026 h(3) h(5)+0.0012547 h(1) h(3)+0.0019085 h(3) h(4) \leq 25$

$h(1)=[78,102]$

$h(2)=[33,45]$

$h(c)=[13,100] \quad c=3,4,5$

$F 3=3 h(1)+0.000001 h(1)^{3}+2 h(2)+0.000002 /\left(3 h(2)^{3}\right) ;$

subject to

$q(1)=h(4)-h(3)+0.550$

$q(2)=h(3)-h(4)+0.550$

$q(3)=1000 \operatorname{scn}(-h(3)-0.25)+1000 \operatorname{scn}(-h(4)-0.25)+894.8-h(1)$

$q(4)=1000 \operatorname{scn}(h(3)-0.25)+1000 \operatorname{scn}(h(3)-h(4)-0.25)+894.8-h(2)$

$q(5)=1000 \operatorname{scn}(h(4)-0.25)+1000 \operatorname{scn}(h(4)-h(3)-0.25)+1294.8$

$h(c)=[0,1200] \quad c=1,2$

$h(c)=[-0.55,0.55] \quad c=3,4$

$F 4=(h(1)-10)^{3}+(h(2)-(20))^{3}$

subject to

$q(1)=(h(1)-5)^{2}+(h(2)-5)^{2}-100 \leq 0$

$q(2)=-(h(1)-6)^{2}-(h(2)-5)^{2}+82.81 \leq 0$

$h(1)=[13,100]$

$h(2)=[0,100]$

$F 5=h(1)^{2}+h(2)^{2}+h(1) h(2)-14 * h(1)-16 h(2)+(h(3)-10)^{2}+4(h(4)-5)+$ $(h(5)-3)^{2}+2(h(6)-1)^{2}+5 h(7)^{2}+7(h(8)-11)^{2}+2(h(9)-10)^{2}+(h(10)-7)^{2}+45$ subject to

$q(1)=105-4 h(1)-5 h(2)+3 h(7)-9 h(8) \geq 0$ 


$$
\begin{aligned}
& q(2)=-3(h(1)-2)^{2}-4(h(2)-3)^{2}-2 h(3)^{2}+7 h(4)+120 \geq 0 \\
& q(3)=-10 h(1)+8 h(2)+17 h(7)-2 h(8) \leq 0 \\
& q(4)=-h(1)^{1}-2(h(2)-2)^{2}+2 h(1) h(2)-14 h(5)+6 h(6) \geq 0 \\
& q(5)=8 h(1)-2 h(2)-5 h(9)+2 h(10)+12 \geq 0 \\
& q(6)=-5 h(1)^{2}-8 h(2)-(h(3)-6)^{2}+2 h(4)+40 \geq 0 \\
& q(7)=3 h(1)-6 h(2)-12(h(9)-8)^{2}+7 h(10) \geq 0 \\
& q(8)=-0.5(h(1)-8)^{2}-2(h(2)-4)^{2}-3 h(5)^{2}+h(6)+30 \geq 0 \\
& h(c)=[-10,10] \quad c=1, . ., 10
\end{aligned}
$$$$
F 6=\operatorname{scn}(2 h(1))^{3}(2 h(2)) /(h(1)(h(1)-h(2)))
$$

\section{subject to}

$q(1)=h(1)+h(2)+1 \leq$

$q(2)=1-h(1)+(h(2)-4)^{2} \leq 0$

$h(1)=[0,10]$

$F 7=(h(1)-10)^{2}+5(h(2)-12)^{2}+h(3)^{4}+3(h(4)-11)^{2}+10 h(5)^{6}+7 h(6)^{2}+h(7)^{4}-$ $4 h(6) h(7)-10 h(6)-8 h(7)$

subject to

$$
\begin{aligned}
& q(1)=127-2 h(1)^{2}-3 h(2)^{4}-h(3)-4 h(4)^{2}-5 h(5) \geq 0 \\
& q(2)=282-7 h(1)-3 h(2)-10 h(3)^{2}-h(4)+h(5) \geq 0 \\
& q(3)=196-23 h(1)-h(2)^{2}+6 h(6)^{2}+8 h(7) \geq 0 \\
& q(4)=-4 h(1)^{2}-h(2)^{2}-2 h(3)^{2}-5 h(6)+11 h(7) \geq 0 \\
& h(c)=[-10,10] \quad c=1, . .7
\end{aligned}
$$$$
F 8=h(1)+h(2)+h(3)
$$

\section{subject to}

$$
\begin{aligned}
& q(1)=1-0.0025(h(4)+h(6)) \geq 0 \\
& q(2)=1-0.0025(h(7)+h(7)-h(4)) \geq 0 \\
& q(3)=1-0.01(h(8)+h(5)) \geq 0 \\
& q(4)=h(1) h(6)-833.33252 h(4)-100 h(1)+83333.333 \geq 0 \\
& q(5)=h(2) h(7)-120 h(5)-h(2) h(4)+1250 h(4) \geq 0 \\
& q(6)=h(3) h(8)-125000-h(3) h(5)+2500 h(5) \geq 0
\end{aligned}
$$


$h(1)=[100,10000]$

$h(c)=[1000,10000] \quad c=2,3$

$h(c)=[10,1000] \quad c=4, . ., 8$

$F 9=h(1)^{2}+(h(1)-1)^{2}$

subject to

$q(1)=h(2)-h(1)^{2}$

$h(c)=[-1,1] \quad c=1,2$

\subsection{Computational Results}

The computational result is given in table Tab 3

Tab 3: Result obtained with CS and ECIS

\begin{tabular}{|c|c|c|}
\hline \hline No & CS & ECIS \\
\hline F1 & -46.00000 & -46.000008 \\
\hline F2 & -40792.14100 & -49744.1214 \\
\hline F3 & 300.47455 & 210.11130 \\
\hline F4 & -7973.0000 & -8881.24000 \\
\hline F5 & -84.00000 & -92.94429 \\
\hline F6 & - inf & -inf \\
\hline F7 & 6.90268 & 2.14460 \\
\hline F8 & 4434.86940 & 4221.91242 \\
\hline F9 & 1.98010 & 5.00000 \\
\hline
\end{tabular}

\section{DISCUSSION ON NUMERICAL RESULTS}

From the result tabulated in table 3.1, it can be seen that ECIS proposed in this paper performs better than CS in terms of value of the objective function in seven test functions out of nine test functions in table 3.1 .

\section{CONCLUSION}

To improve the performance of CS algorithm, an enhanced CS algorithm ECIS algorithm based on calculating the CS parameters at each iteration is introduced in this paper. In conclusion, the ECIS algorithm outperforms standard CS algorithms in term of reaching optimum solution, speed and robustness. Therefore ECIS algorithm is recommended for solving optimization problems. 


\section{Conflit of Interest}

The authors declare no conflict of interest

\section{Data Avaliability}

Not applicable

\section{REFERENCES}

[1] Yang, X-S. \& Deb, S. (2009). Cuckoo search via levy flight. Nature and Biologically Inspired Computing(NaBIC) World Congress on Nature and. Biologically Inspired Computing.

[2] Anuja, S.J., Omkar, K., Kakandikar, G. M. \& Nandedkar, V.M. (2016). Cuckoo search optimization - a review. International Conference on Advancements in Aeromechanical Materials for Manufacturing.

[3] Sanusi, Y. A., Muhammed, B. M., Sani M., Zaharudden, H. Salawudeen, A. T. \& Prosper, O. (2019). Development of a dynamic cuckoo search algorithm. Covenant Journal of Information and Communication Technology, 7 .

[4] Erik, C. \& Adolfo, R. (2014). A cuckoo search algorithm for multimodal optimization.The Scientific World Journal.Article ID 497514

[5] Ehsan, V., Shahran, M. \& Saheed, T. (2011). Improved cuckoo search algorithm for global optimization. International Journal of Communication and Information Technology, 1.

[6] Venkata, V. G. \& Ravi K. V. (2016).Cuckoo search optimization and its applications: a review. International Journal of Advanced Research in Computer and communication Engineering, 5(11).

[7] Liping, L., Ning, W. \& Peijun, Z. (2018). Modified cuckoo search algorithm with variational parameters and logistic map. MDPI Journal.

[8] Aina, I. I. \& Ejieji, C.N. (2020). Refined heuristic swarm intelligence algorithm. Earthline Journal of Mathematical Sciences, 5(2), 267-275. https://doi.org/10.34198/ejms.5221.267

[9] Akinsunmade, A. E., \& Aina, I. I. (2020). Water distribution network design using hybrid self-adaptive multi-population elitist pollination intelligence (HSAMPEPI) jaya algorithm. Earthline Journal of Mathematical Sciences,5(2), 329-343.

https://doi.org/10.34198/ejms.5221.329343 
[10] Koziel, S. \& Michalewicz, Z. (1999). Evolutionary algorithms, homomorphous mapping, and constrained parameter optimization problem. Evolutionary Computation,7 (19-44). 
